(C) 1999 IEEE. Personal use of this material is permitted. Permission from IEEE must be obtained for all other uses, in any current or future media, including reprinting/republishing this material for advertising or promotional purposes, creating new collective works, for resale or redistribution to servers or lists, or reuse of any copyrighted component of this work in other works. 


\title{
Initial Results from a PET/Planar Small Animal Imaging System
}

\author{
S. Siegel ${ }^{1}$, Member, IEEE, J.J. Vaquero, Member, IEEE, L. Aloj ${ }^{2}$, J. Seidel, E. Jagoda ${ }^{3}$, W.R. \\ Gandler ${ }^{4}$, Senior Member, IEEE, W.C. Eckelman ${ }^{3}$, M.V. Green \\ Nuclear Medicine Department, ${ }^{3}$ PET Department \\ ${ }^{4}$ Center for Information Technology \\ National Institutes of Health, Bethesda, Maryland, 20892
}

\begin{abstract}
A pair of stationary, opposed scintillation detectors in time coincidence is being used to create planar projection or tomographic images of small animals injected with positronemitting radiotracers. The detectors are comprised of arrays of individual crystals of bismuth germanate coupled to position-sensitive photomultiplier tubes. The system uses FERA (LeCroy Research Systems) charge-sensitive ADCs and a low cost digital $\mathrm{V} / \mathrm{O}$ board as a FERA bus-to-host bridge. In projection mode, the animal is placed within the $55 \mathrm{~mm} \mathrm{x}$ $45 \mathrm{~mm}$ useful field-of-view of the detectors and images are formed from coincidence lines that fall close to the normals of both detectors. In tomographic mode, the animal is placed on a rotation stage between the detectors and rotated around a vertical axis to acquire all possible lines-of-response. Tomographic images are then reconstructed from those lines falling within a user-specified angle of each detector normal. In mice, the system is capable of high-speed, whole-body dynamic projection imaging, and whole body tomographic imaging of slowly varying tracer distributions. An ECG gating capability is also available for evaluating cardiac function. This system is currently being used to study tracer transport in normal and genetically engineered mice.
\end{abstract}

\section{INTRODUCTION}

Rodent models of human diseases and animals with genetic alterations affecting virtually every organ system are increasingly common [1]-[5]. Accompanying the creation of these models is the need to characterize a disease process and changes in that process that may occur in response to therapy, or to characterize often subtle and long-term phenotype variations induced by genetic manipulation. Nuclear medicine radiotracer techniques are ideally suited for both of these tasks since these methods can be directed toward specific biochemical pathways and are, in general, non-invasive. Nuclear medicine imaging systems developed for human use, however, do not possess sufficient spatial resolution and sensitivity to accurately quantify dynamically changing organ radioactivity in animals the size of the mouse. In response to these limitations, a number of laboratories have begun developing high performance PET imaging systems specifically for imaging small animals [6]-[10]. In this report, we describe an imaging system developed in our laboratory for the purpose of imaging mice and rats that provides substantial functionality at moderate cost.

Current addresses:

${ }^{1}$ Concorde Microsystems, 10427 Cogdill Rd, Suite 500, Knoxville, Tennessee 37932

${ }^{2}$ Centro per la Medicina Nuclear, Consiglio Nazionale delle

Ricerche, Via S. Pansini 5, Napoli, Italy.

\section{MATERIALS AND METHODS}

\section{A. System Characteristics}

The general layout of the system is shown in Figure 1. Each detector consists of a $26 \times 22$ array of bismuth germanate (BGO) crystals coupled with optical grease to the face of a Hamamatsu R3941 position-sensitive photomultiplier tube (PSPMT) [11], [12]. Each crystal is $2 \mathrm{~mm} \times 2 \mathrm{~mm} \times 10 \mathrm{~mm}$ long, polished on five sides and finely ground on their entrance end. All crystals are double-wrapped on their long sides with PTFE tape including the entrance end [9]. In all of the experiments described below, the detectors were $18 \mathrm{~cm}$ apart.

Tomographic imaging is performed with the detector pair horizontal as shown in Figure 1. The object to be imaged is attached to a rotation device placed midway between the detectors. During data acquisition, the object is rotated around a vertical axis to acquire all possible lines-of-response. Tomographic images are then reconstructed from lines that fall within a user-selected range of angles relative to the detector normals.

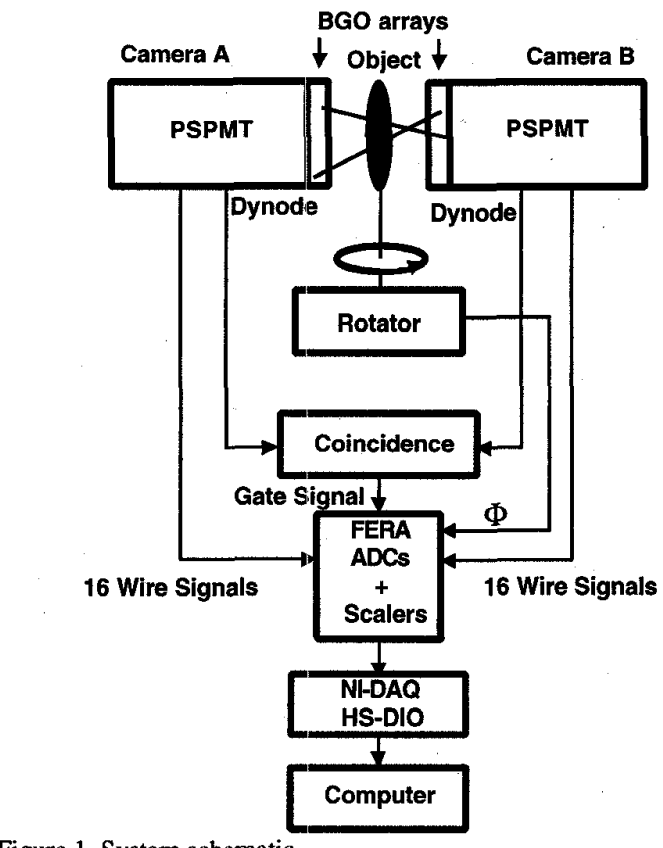

Figure 1. System schematic. 
Planar projection imaging is performed by mechanically rotating the detector pair 90 degrees into a vertical orientation (not shown) and placing the object to be imaged on a small table inserted between the detectors.

The R3941 possesses $18 \mathrm{X}$ anode wires and $16 \mathrm{Y}$ anode wires. In order to reduce the number of these signal lines from 34 to 16 , the $X$ wires were combined by pairs except for the end wires which were combined in threes. The $Y$ wires were combined by pairs, yielding a total of 32 signal lines (two FERA modules) for the combined tubes. In addition to these two FERA modules, a LeCroy 2366 universal logic module was programmed to be FERA compatible and to act as a three channel, 32 bit, $40 \mathrm{MHz}$ scaler. This scaler serves to count time ticks during dynamic planar studies and/or rotation steps during tomographic imaging. It also keeps track of the number of detected and the number of processed events. A trigger signal is derived from the last dynode of each tube and used for coincidence detection. Detection of a coincidence event initiates digitization of all 32 amplified and delayed signals from both detectors, and readout of the scalers.

To circumvent the bandwidth limitation of the CAMAC Dataway [13], we assembled a FERA-compatible, differential ECL-to-TTL level translator to interface the FERA bus to an inexpensive, off-the-shelf digital I/O board (National Instruments PCI-DIO-32HS) [14]. This board was chosen because it provides six different modalities of hardware handshaking, two independent 16 bit wide channels, a maximum $80 \mathrm{MB} / \mathrm{s}$ bandwidth and bus master scatter-gather DMA. In our implementation, we used the 8255 emulation handshake mode running on a $300 \mathrm{MHz}$ Pentium II host computer (Figure 2).

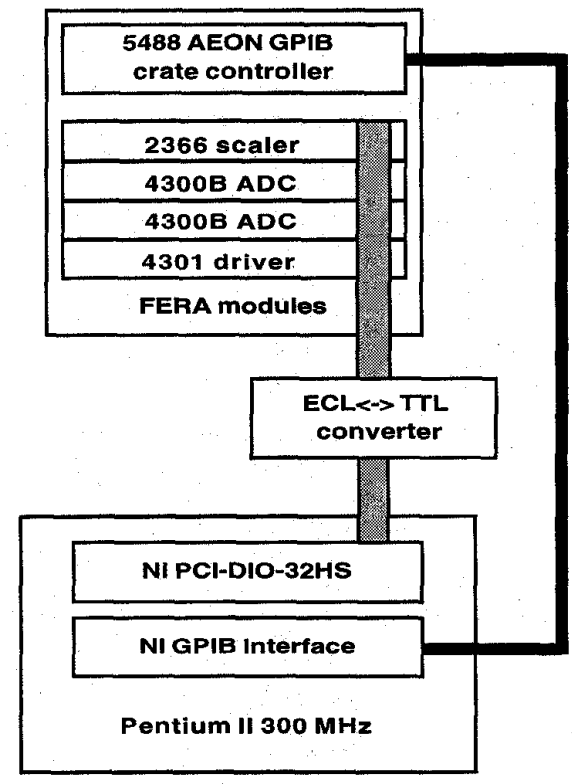

Figure 2. Data acquisition system schematic.
The host also initializes and configures the CAMAC modules via the GPIB controller and starts the acquisition, which is either terminated by reaching a preset time condition or by the user.

Currently, no real time processing has been implemented except for an optional lossless data compression that reduces the amount of disk space needed to store the list mode file.

\section{B. Performance Characteristics}

In order to assess the overall effectiveness of crystal/PSPMT coupling, spatial linearity, and the appearance of crystals near the edges of the field-of-view, the detectors (in time coincidence) were illuminated with $511 \mathrm{keV}$ radiation from a volume ${ }^{18} \mathrm{~F}$ source placed between them.

Tomographic resolution (FWHM=2.1 $\mathrm{mm}$ ) was measured by imaging two parallel ${ }^{18} \mathrm{~F}$ filled capillary tubes separated by $7.1 \mathrm{~mm}$ as they were rotated around a vertical axis midway between the detectors.

Central point source sensitivity without energy thresholding $(130 \mathrm{cps} / \mu \mathrm{Ci})$ was measured by placing a small, low activity ${ }^{18} \mathrm{~F}$ source at the geometric center of the system and measuring the true coincidence count rate.

The appropriate prompt coincidence window (12 ns) was determined by placing an ${ }^{18} \mathrm{~F}$ source midway between the two detectors and connecting the outputs of each discriminator to the inputs of a time-to-amplitude converter. The time axis was calibrated by inserting a delay of known length into one of these lines and repeating the experiment. Time resolution determined in this manner was $6 \mathrm{~ns}$ full-width-half-maximum.

Count rate performance was assessed by monitoring the prompt and random coincidence event rates during the decay of a ${ }^{18} \mathrm{~F}$ source of known activity placed in between the detectors. Random rates were obtained by applying a time shift of $50 \mathrm{~ns}$ to the coincidence window. Multiplicative dead time correction factors were calculated as the ratio of detected rate to processed coincidence rate. Both numbers were supplied by the scaler module.

\section{Rat and Mouse Studies}

Two kinds of animal imaging studies were selected to illustrate the use of this system. In the first, a 300 gram rat was injected with ${ }^{18} \mathrm{~F}$ fluoride which readily accumulates in the skeleton. At the end of the 30 minutes uptake period, the animal was sacrificed, attached to the rotation device so that the upper half of the animal was in the field-of-view, and imaging began. Upon completion of this acquisition, the translation stage was advanced upward such that the second acquisition visualized the lower half of the animal. These image data were reconstructed using conventional filtered backprojection and then fused to form a complete whole body scan of the animal's skeleton.

The second study was performed in a small normal mouse after configuring the detector pair for planar imaging as described above. The anaesthetized animal was placed on the imaging table, centered within the field-of-view, injected with ${ }^{18} \mathrm{~F}$ FDG and imaging immediately began. Image data were acquired for 20 minutes and then binned into sequential 10 second frames. This image sequence was analyzed to 
visualize the transport of tracer throughout the animal and to quantify the time variation of radioactivity in various organs.

\section{RESULTS}

\section{A. Detectors}

Flood images with the two detectors in coincidence are shown in Figure 3. All of the individual crystals in one of the detectors (A in Figure 3 ) are detectable, while one column of crystals is missing from the second detector and not all of the remaining columns are fully resolved. These effects are most likely due to the BGO array being slightly offset from the effective FOV of the PSPMT. In the current design, the array and PSPMT FOV cannot be realigned once the module is assembled.

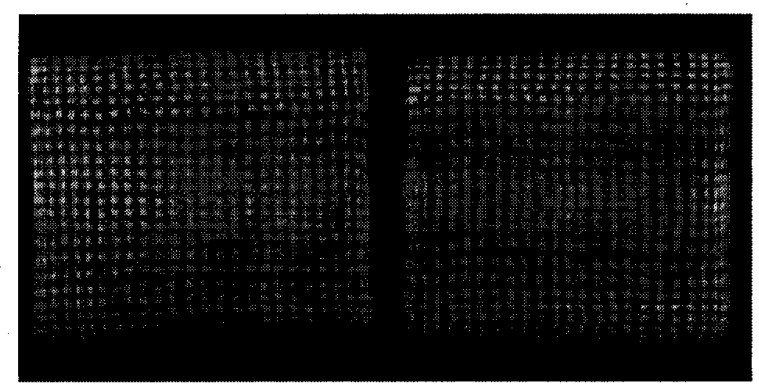

A

B

Figure 3. Field flood illumination of the detector pair with a volume distribution of ${ }^{18} \mathrm{~F}$ and the detectors in time coincidence. All $26 \times 22$ crystals are visible in detector A, but only $25 \times 22$ in detector B since the two first columns are not resolved separately.

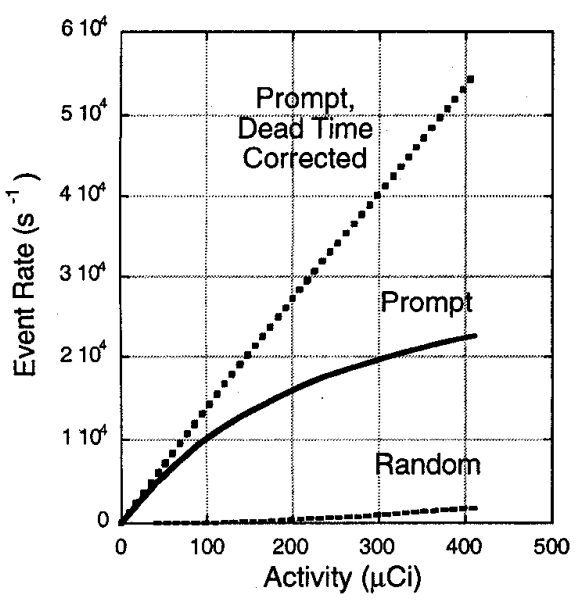

Figure 4. Measured system performance as a function of F-18 source activity.

\section{B. Data Acquisition System}

The I/O board operated in 8255 emulation mode sustained a data throughput of $3.4 \mathrm{MB} / \mathrm{s}$. This value corresponds to acquiring $>45,000$ events/s $(5 \mu$ s for the $\mathrm{A} / \mathrm{D}$ conversion plus
$17.2 \mu$ s for transferring to the host), each event consisting of 38 words ( 76 bytes)

Figure 4 shows the measured prompt and random event rates as a function of ${ }^{18} \mathrm{~F}$-source activity between the two detectors, as well as the dead time-corrected prompt event rate. The dead time-corrected event rate follows a straight line, as it should.

\section{Rat and Mouse Studies}

The whole body bone scan of the rat injected with ${ }^{18} \mathrm{~F}$ fluoride is shown in Figure 5. The upper panel (A) contains reprojections of the tomographic data into posterior and lateral projections. Selected tomographic slices are shown in panel (B).
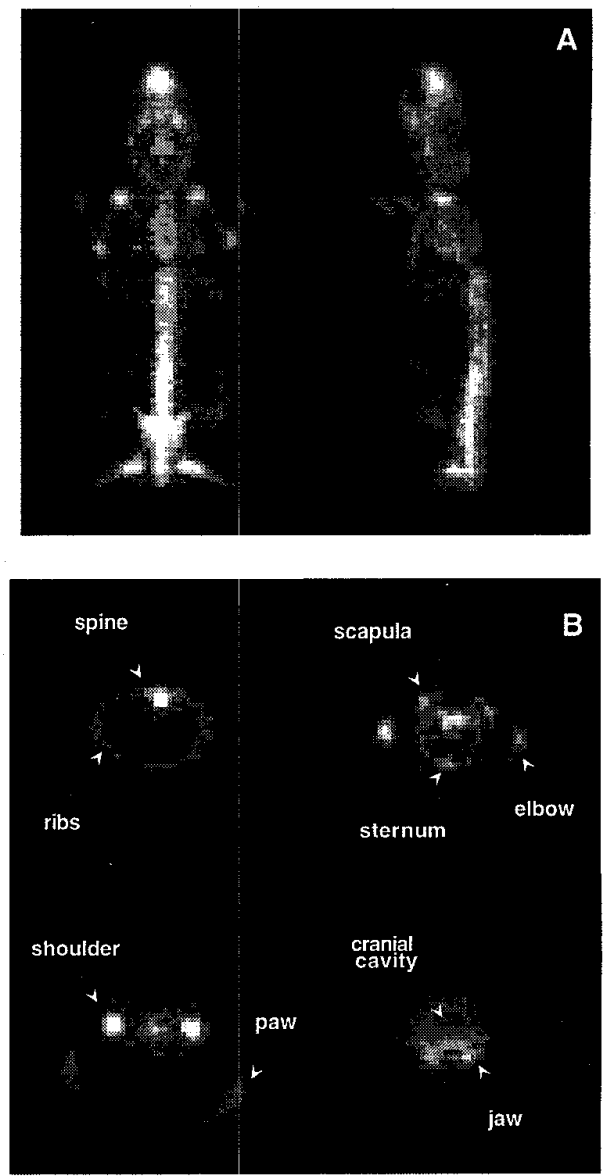

Figure 5. (A) Posterior (left), left lateral (right) reprojection images of the rat skeleton. (B) Selected tomographic images from the same study.

Selected images from the dynamic, planar projection image sequence acquired in the normal mouse are shown in Figure 6. In these images, the animal is viewed in posterior projection with the nose at the top of each figure, the tail at the bottom, and the animal's right to the observer's right. The bright central structure in the 10 second image is the heart. 
The bright object at the bottom of the 20 minute image is the bladder. Time-activity curves created from ROIs over the heart, liver, left kidney and bladder are shown at the bottom of the figure. These curves were corrected for dead time, radioactive decay, injected dose and ROI size.
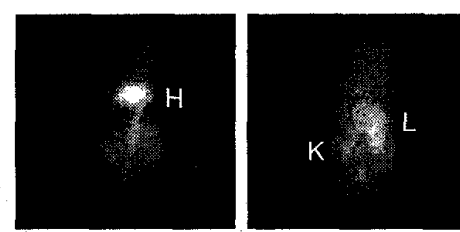

$2 \min$

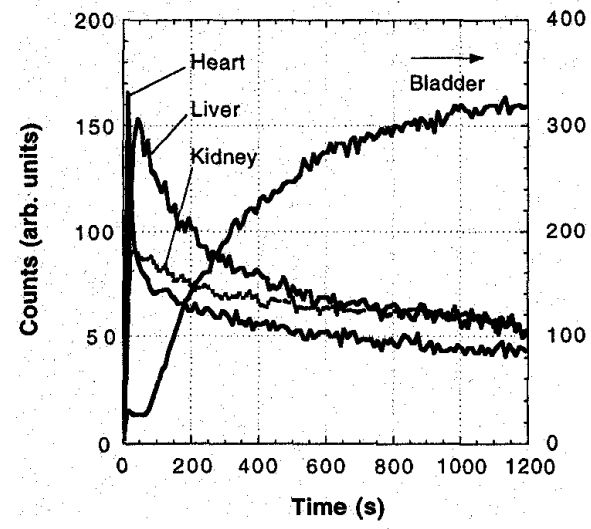

Figure 6. Upper panels: selected images following tail vein injection of ${ }^{18} \mathrm{~F}$ FDG into a normal mouse. Lower panel: time-activity curves over the liver $(\mathrm{L})$, heart $(\mathrm{H})$, left kidney $(\mathrm{K})$ and bladder $(\mathrm{B})$.

\section{DISCUSSION}

Although this PET/planar imaging system is comprised of only two detectors and requires mechanical rotation of the animal to obtain tomographic images, a wide variety of studies can be carried out with this device. Planar projection imaging of mice (Figure 6), for example, allows nearly the whole body of a mouse to be viewed at once so that organ radioactivity at multiple sites throughout the animal can be assessed at the same moment. Such knowledge, suggested by the timeactivity curves shown in Figure 6 , should present novel opportunities for modeling the transport of new radiopharmaceuticals and changes in organ function due to genetic or other manipulations. While projection imaging cannot match the absolute quantitative accuracy of tomographic imaging, the use of background corrected timeactivity curves can provide approximate, high temporal resolution information that can, in many cases, answer the question at hand.

The tomographic reprojections obtained in the rat suggest that, for animals this size, a spatial resolution near $2 \mathrm{~mm}$ is sufficient to reveal considerable detail, at least for high intrinsic contrast tracers such as ${ }^{18} \mathrm{~F}$ fluoride. The ribs, sternum, spine, pelvis, skull and small bones of the skull are all evident in these images, as well as the metabolically active ends of the long bones.

While the present device possesses useful capabilities, a host of modifications can be envisioned that would improve performance significantly. Replacement of the BGO arrays with LSO or LGSO or some combination of other fast, high stopping power scintillators [15]-[18] would increase position detection accuracy and reduce pulse pileup and random coincidences at high rates.

Preliminary experiments suggest that fast synchronous transfer ("burst mode") can double data throughput for the existing system. Even greater throughput could be achieved if the number of signals defining an event is reduced (by resistive charge division of the anode signals). Implementation of an expectation maximization reconstruction algorithm with proper system model would also be expected to significantly enhance system performance [19].

\section{CONCLUSION}

High-speed planar projection imaging and rotational tomography of slowly varying tracer distributions, useful subsets of all possible imaging studies, can be performed with a relatively simple and inexpensive pair of BGO detector modules in time coincidence.

The study also suggests that the digital I/O board approach may provide a high speed, low cost option for FERA data acquisition.

\section{ACKNOWLEDGMENTS}

The authors thank Burt Chidakel for his support in producing the level translators and the implementation of the rotator hardware for the tomographic acquisitions. S. Siegel acknowledges the support of the National Research Council and thanks them for providing the mechanism by which he arrived at the National Institutes of Health. J.J. Vaquero was supported, in part, by a grant from CICYT (Spanish Government).

\section{REFERENCES}

[1] M.R. Capecchi, "Targeted gene replacement", Sci Amer (Intl Ed), vol. 270 (no. 3), pp. 34-41, March 1994.

[2] M.M. Humphries, D. Rancourt, G.J. Farrar, P. Kenna P, et al., "Retinopathy induced in mice by targeted disruption of the rhodopsin gene", Nat Gen, vol. 15 (no. 2), pp. 216219, February 1997.

[3] H. Matsumoto, A. Takahashi, X. Wang, K. Ohnishi, et al., "Transfection of p53 knockout mouse fibroblasts with wildtype p53 increases the thermosensitivity and stimulates apoptosis induced by heat stress", Intl J Rad Onc Bio Phys, vol. 39 (no. 1), pp. 197-203, August 1997.

[4] B.G. Condie, G. Bain, D.I. Gottlieb, M.R. Capecchi, "Cleft palate in mice with a targeted mutation in the gamma-aminobutyric acid-producing enzyme glutamic 
acid decarboxylase 67", Proc Natl Acad Sci USA, vol. 94 (no. 21), pp. 11451-11455, October 1997.

[5] E.M. Carpenter, J.M. Goddard, A.P. Davis, T.P. Nguyen, M.R. Capecchi, "Targeted disruption of Hoxd-10 affects mouse hindlimb development", Devel, vol. 124 (no. 22), pp. 4505-4514, November 1997.

[6] S. Weber, A. Terstegge, H. Herzog, et al., "The Design of an Animal PET: Flexible Geometry for Achieving Optimal Spatial Resolution or High Sensitivity", IEEE Trans Med Imag, vol. 16 (no. 5), pp. 684-689, October 1997.

[7] C.J. Marriott, J.E. Cadorette, R. Lecomte, V. Scasnar, et al., "High-resolution PET imaging and quantitation of pharmaceutical biodistributions in a small animal using avalanche photodiode detectors", $J$ Nucl Med, vol. 35, pp. 1390-1396, August 1994.

[8] P.V. Kulkarni, P.P. Antich, A. Constantinescu, J. Prior, et al., "Folate receptor imaging with ${ }^{125}$ I labeled folic acid with a whole body small animal imaging device built with plastic scintillating optical fibers", Nucl Instr Meth B, vol. B99, pp. 800-803, May 1995.

[9] S.R. Cherry, Y. Shao, R.W. Silverman, K. Meadors, et al., "MicroPET: A high resolution PET scanner for imaging small animals", IEEE Trans Nucl Sci, vol. 44 (no. 3), pp. 1161-1166, June 1997.

[10] A.G. Weisenberger, E.L. Bradley, S. Majewski, M.S. Saha, "Development of a novel radiation imaging detector system for in vivo gene imaging in small animal studies", IEEE Trans Nucl Sci, vol. 45 (no. 3), pp. 1743-1749, June 1998.

[11] J. Seidel, W.R. Gandler, I.N. Weinberg, A. Markowitz, N.J. Lobaugh, M.V. Green, "Collimatorless Projection Imaging of Positron Emitters with an Opposed Pair of BGO Scintillation Cameras", $J$ Nucl $\mathrm{Med}$, vol. 36, pp. 177P, May 1995.

[12] J. Seidel, C. Johnson, E. Jagoda, L. Sha, W. C. Eckelman, M.V. Green, "A High Resolution Scanner for Investigating Technical Requirements and Selected Biological Applications of Continuous 3D Detectors in Small Animal PET", J Nucl Med, vol. 38, pp. 44P, May 1997.

[13] CAMAC Instrumentation and Interface Standards, IEEE, 1982.

[14] DIO 6533 User Manual, National Instruments, 1997.

[15] C. L. Melcher and J. S. Schweitzer, "Cerium-doped Lutetium Oxyorthoslicate: A Fast, Efficient New Scintillator," IEEE Transaction on Nuclear Science, vol. 39, pp. 502-505, 1992.

[16] M. E. Casey, L. Eriksson, M. Schmand, M. S. Andreaco, M. Paulus, M. Dahlbom, and R. Nutt, "Investigation of LSO Crystals for High-Spatial-Resolution Positron Emission Tomography," IEEE Transactions on Nuclear Science, vol. 44, pp. 1109-1113, 1997.

[17] F. Daghighian, P. Shenderov, K. S. Pentlow, M. C. Graham, B. Eshaghian, C. L. Melcher, and J. S. Schweitzer, "Evaluation of Cerium Doped Lutetium Oxyorthosilicate (LSO) Scintillation Crystal for PET," IEEE Transactions on Nuclear Science, vol. 40, pp. 10451047, 1993.
[18] R. Lecomte, C. Pepin, D. Rouleau, A. Saudi, M.S. Andreaco, M. Casey, R. Nutt, H. Dautet, P.P. Webb, "Investigation of GSO, LSO and YSO Scintillators Using Reverse Avalanche Photodiodes", IEEE Trans Nucl Sci, vol. 45, pp. 478-482, June 1998.

[19] C.A. Johnson, J. Seidel, R.E. Carson, W.R. Gandler, et al., "Evaluation of $3 \mathrm{D}$ reconstruction algorithms for a small animal PET camera", IEEE Trans Nucl Sci, vol. 44, pp. 1303-1308, June 1997. 